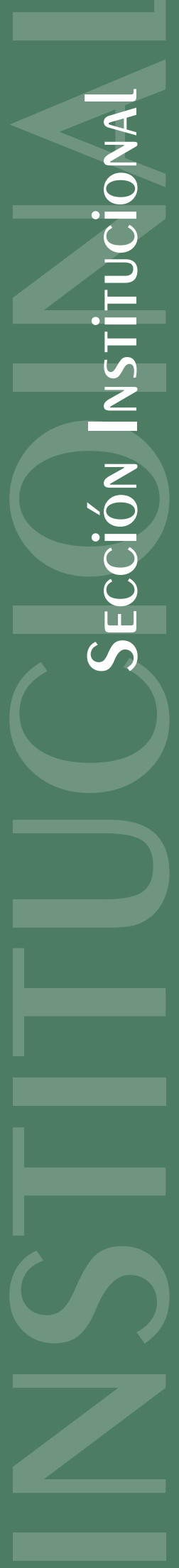




\title{
LA CELEBRACIÓN DE LOS OCHENTA AÑOS DE LA EDUCACIÓN FÍSICA COMO PROFESIÓN EN COLOMBIA. EL MOTOR PARA RETOMAR UN TRABAJO INVESTIGATIVO
}

\author{
EIGHTY YEARS OF PHYSICAL EDUCATION AS A PROFESSION IN COLOMBIA. AN \\ ENCOURAGEMENT TO RESUME RESEARCH WORK
}

\section{A CELEBRAÇÃO DOS OITENTA ANOS DA EDUCAÇÃO FÍSICA COMO PROFISSÃO NA COLÔMBIA. O MOTOR PARA RETOMAR UM TRABALHO DE PESQUISA}

Germán Preciado Mora ${ }^{1}$

\section{Resumen}

\begin{abstract}
Este documento reseña los principales eventos que se han desarrollado desde el sábado 25 de junio de 2016 hasta los primeros meses del 2017 como parte de la celebración del octogésimo aniversario de acción pedagógica profesional de la Educación Física en Colombia. Este artículo se enfoca en las actividades realizadas desde la génesis de la gala inaugural, pasando por la gran muestra fotográfica de los ochenta años de la educación física colombiana, el conversatorio "Pasado-presente y futuro de la educación física en Colombia", y otros eventos y actividades dentro del año de celebración. Con ello, este artículo busca contribuir al reconocimiento social y académico de una carrera que ha formado en estos ochenta años a miles de profesionales que han estado al servicio del país y de su población desde el 25 de junio de 1936, cuando, por medio de la promulgación del Decreto 1528, se establece la formación profesional de Profesores de Educación Física en Colombia.
\end{abstract}

Palabras clave: formación de profesores; educación física; historia; Colombia

\section{Abstract}

This document outlines the main events that have taken place since Saturday, June 25, 2016 and until the first months of 2017, as part of the celebration of the eightieth anniversary since Physical Education became a profession in Colombia. This article focuses on the activities carried out since the beginning of the opening ceremony, moving through the great photographic exhibition of the eighty years of Colombian physical education, the round table on "Past-present and future of physical education in Colombia," and other events and activities held over the year of celebration. This article seeks to contribute to the social and academic recognition of a career that has trained, over the past eighty years, thousands of professionals who have been at the service of the country and its people since June 25, 1936, when the professional training of physical education teachers in Colombia became official through the enactment of Decree 1528.

Keywords: teacher training; physical education; history; Colombia

* Licenciado en Educación Física Universidad Pedagógica Nacional. Magíster en Administración Universidad de la Salle. Docente Investigador de la Secretaría de Educación de Bogotá. Gerente de Gerpremo Inversiones. Leader Coach del Nodo Actividad Física y Cuerpo de la Red Distrital de Docentes Investigadores REDDI y de la RED Local de Educación Física de Kennedy. Promotor y practicante activo de la Educación Física Mental y del Walking Life. Correo electrónico: gerpremo@yahoo.es, gerpremo@gmail.com 
Este documento resenha os principais eventos desenvolvidos desde o sábado 25 de junho de 2016 até os primeiros meses do 2017 como parte da celebração do octogésimo aniversário de ação pedagógica profissional da Educação Física na Colômbia. Este artigo faz ênfase nas atividades realizadas desde a gênese da gala inaugural, passando pela grande mostra fotográfica dos oitenta anos da educação física colombiana, a conversação "passado-presente e futuro da educação física na Colômbia", e outros eventos e atividades dentro do ano de celebração. Com o anterior, este artigo visa contribuir ao reconhecimento social e acadêmico de um programa que já formou milhares de profissionais nestes oitenta anos, que têm contribuído ao país e sua população desde o dia 25 de junho de 1936, quando o Decreto 1528 estabeleceu a formação profissional de Professores de Educação Física na Colômbia.

Palavras chave: formação de professores; educação física; história; Colômbia

Fecha de recepción: 27 de julio de 2016

Fecha de aprobación: 19 de febrero de 2017

Para citar este artículo:

Preciado, G. (2017). La celebración de los 80 años de la educación física como profesión en Colombia. El motor para retomar un trabajo investigativo. Lúdica Pedagógica, (25), 159-172. 


\section{INTRODUCCIÓN}

La celebración de los 80 años como profesión que cumpliría la Educación Física colombiana fue el motor que impulso al Nodo Actividad Física y Cuerpo de la Red Distrital de Docentes Investigadores (REDDI) ${ }^{2}$ para retomar el trabajo investigativo que se había desarrollado en el Grupo de Investigación Rescate Histórico de la Educación Física, el Deporte y la Recreación, de la Facultad de Educación Física de la Universidad Pedagógica Nacional ${ }^{3}$.

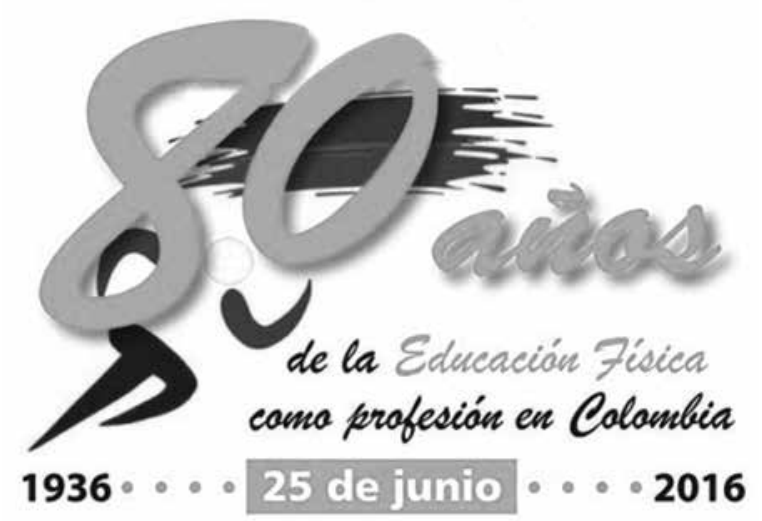

Logo Oficial de la Celebración. Propiedad del Comité Interinstitucional de Conmemoración de los 80 años de la Educación Física como profesión en Colombia.

2 El nodo Actividad Física y Cuerpo lo conforman Docentes de Educación Física, Deporte, Recreación y Profesionales de Ciencias Afines, que abierta y públicamente manifiestan su interés y compromiso por pertenecer y aportarle al Nodo y a la Red Distrital de Docentes Investigadores (REDDI), en busca de desarrollo personal, profesional, del saber disciplinar y de brindar una educación de calidad a partir de procesos investigativos y de innovación para los niños, niñas y jóvenes de Bogotá y Colombia. Actualmente, este Nodo está liderado por los profesores Ingrid Nathalia Meneses Runza y Germán Preciado Mora.

3 El Grupo de Investigación Rescate Histórico de la Educación Física, el Deporte y la Recreación de la Facultad de Educación Física de la Universidad Pedagógica Nacional se creó en el año 1999 por iniciativa del profesor Ángel Humberto Vaca Hernández y desarrolló su labor hasta el año 2008, en los siguientes proyectos investigativos: Rescate histórico del saber de la educación Física en la Universidad Pedagógica Nacional; Memoria oral y configuración de la Educación Física en Colombia entre 1936 y 1970; Fotografía y fuentes documentales escritas en la configuración de la Educación Física en Colombia entre 1936 y 1970, aprobados por el Centro de Investigaciones de la Universidad Pedagógica (Ciup) para las vigencias 2000, 2004-2005-20062007 y 2008.

Estuvo integrado en su tiempo de funcionamiento, aunque no simultáneamente, por los profesores: Ángel Humberto Vaca Hernández (+), Raúl Eduardo Bernal Sánchez, Darío Ramírez Duarte (+), Fredy Amazo, Ximena Herrera, Mario Gildardo Loaiza Padilla, José Olegario Rincón Velandia, Olga Cecilia Muñoz Rojas y Germán Preciado Mora.
El Grupo de Investigación Rescate Histórico de la Educación Física, el Deporte y la Recreación, durante el tiempo de funcionamiento y en su trabajo investigativo, recopiló una serie de documentos, fotografías, audios y videos, que hasta el momento, en su mayoría, no se han compartido con la comunidad académica de la Educación Física y que son un material de primera mano para continuar develando el recorrido octogenario que ha hecho la Educación Física, desde aquel jueves 25 de junio de 1936 y llegando hasta hoy, año 2017. Es por esto que el Nodo Actividad Física y Cuerpo de la Red Distrital de Docentes Investigadores (REDDI) asumió la tarea de presentarlos a la comunidad académica, aprovechando esta importante efeméride de nuestra profesión.

\section{LA GÉNESIS DE UNA CELEBRACIÓN}

El Nodo Actividad Física y Cuerpo de la REDDI, desde mediados del año 2015, determina abanderar una propuesta de celebración para la Educación Física, que estableció como objetivo:

Rendir un sentido homenaje a la Educación Física Colombiana en los 80 años de Ejercicio Profesional (junio 25 de 1936-junio 25 de 2016), a través de una serie de eventos académicos, deportivos, recreativos, de educación física, culturales y sociales a nivel local, distrital y nacional con la participación y apoyo de diferentes entidades públicas y privadas de los sectores: educativo, de la actividad física, de las artes y la cultura de Colombia ${ }^{4}$.

Se propusieron, entre otros, los siguientes eventos:

- Eventos académicos distritales y nacionales.

- Gran muestra fotográfica y video sobre el recorrido y desarrollo histórico que ha tenido la Educación Física en ochenta años de ejercicio y acción profesional en beneficio de los niños, jóvenes y adultos colombianos ${ }^{5}$.

- Gala de celebración.

4 Documento de Propuestas para la celebración de los 80 años de la Educación Física como profesión en Colombia, elaborado por el Nodo Actividad Física y Cuerpo de la Red Distrital de Docentes Investigadores (REDDI).

5 Aprovechando las fotografías y videos que recopiló y conserva el profesor Germán Preciado Mora como integrante en su momento del Grupo de Investigación Rescate Histórico de la Educación Física, el Deporte y la Recreación de la Facultad de Educación Física de la Universidad Pedagógica Nacional y ahora como líder del Nodo Actividad Física y Cuerpo de la REDDI. 


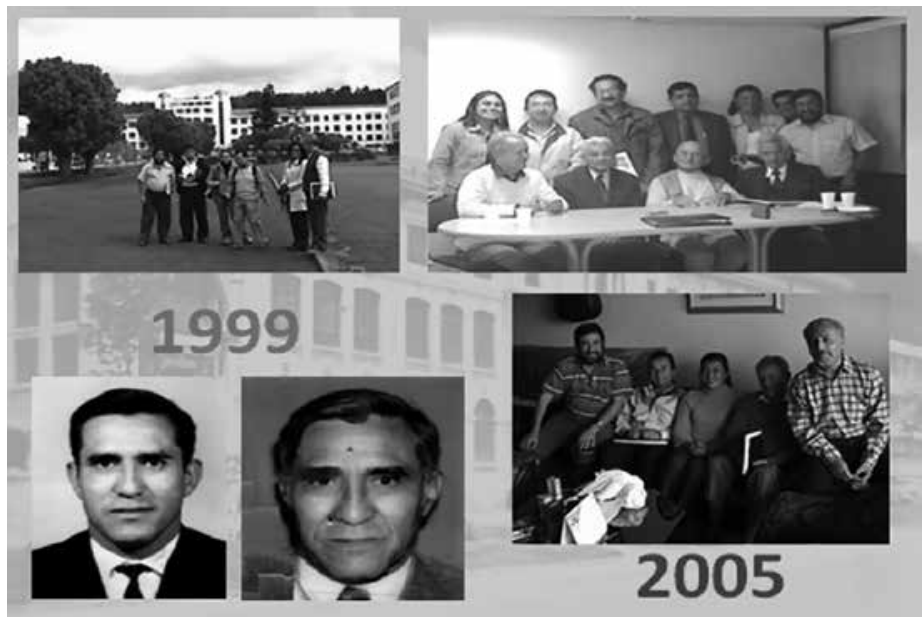

El Grupo de Investigación Rescate Histórico de la Educación Física, el Deporte y la Recreación de la Facultad de Educación Física de la Universidad Pedagógica Nacional, creado en el año 1999 por iniciativa del profesor Ángel Humberto Vaca Hernández (+).

Fuente: Foto Archivo del Grupo de Investigación Rescate Histórico de la Educación Física, el Deporte y Recreación.

- Ceremonia de exaltación y reconocimiento a pioneros y personalidades de la educación física colombiana.

- Walking Life Nacional. Caminata familiar de escolares y sus familias en un recorrido de 8 kilómetros (1 kilómetro por cada década), desarrollada simultáneamente en todo el país por las principales vías de cada municipio y ciudad con llegada a la plaza central, donde además se haría un festival de experiencias exitosas en educación física. Actividad organizada y liderada por los docentes de educación física de cada municipio, con el apoyo de los estudiantes de educación física de las universidades con sedes en la región.

- Recopilación en un documento único (enciclopedia impresa y/o digital) de documentos publicados e inéditos sobre la historia de la educación física colombiana.

- Creación de un museo (físico y/o virtual) de historia de la educación física colombiana donde se puedan exhibir los documentos (escritos, fotográficos, audiovisuales, de prensa) y materiales propios y de ayuda para los procesos educativos de la educación física, para que sirva de material de consulta y de reconocimiento histórico para toda la comunidad educativa e investigativa de Colombia y el mundo.

Con el norte trazado, los líderes del Nodo Actividad Física y Cuerpo de la REDDI comienzan a gestionar la implementación y desarrollo de estos eventos, a partir de diálogos con personas, profesionales y entidades del medio con las que se fueron estableciendo alianzas y acuerdos de ayuda mutua para su realización, en un recorrido que incluyó las siguientes acciones, tareas, eventos y convenios que se presentan a continuación, teniendo en cuenta el orden cronológico de desarrollo.

\section{LA GRAN MUESTRA FOTOGRÁFICA OCHENTA AÑOS DE LA EDUCACIÓN FÍSICA COLOMBIANA}

Aprovechando el material fotográfico y audiovisual con que se cuenta, se planea la organización y desarrollo de la gran muestra fotográfica "Ochenta años de la educación física colombiana", pensada como una exposición itinerante que se exhibiera en varios eventos académicos y en las instalaciones de diferentes entidades públicas y privadas del sector educativo, de la actividad física y de las artes de Colombia, para lo cual se invitó públicamente y a través de las redes a la comunidad académica de la Secretaría de Educación de Bogotá y de la Actividad Física y las Artes de Bogotá y de Colombia a participar enviando sus fotografías.

Finalmente, la gran muestra fotográfica "Ochenta años de la educación física" se construyó en dos momentos, gracias a la alianza entre la Red Local de Educación Física de Kennedy, el Nodo Actividad Física y Cuerpo de la REDDI y el Centro Virtual de Memoria en Educación y Pedagogía del IDEP, y quedó compuesta por un total de 50 fotografías que permiten al observador transportarse al pasado, con el ánimo de reflexionar y conocer sobre el devenir de nuestra profesión. En el primer momento, se 
exhibieron 25 fotografías que mostraron al público los usos y concepciones del cuerpo en los ochenta años de acción pedagógica de la educación física colombiana; a partir del 16 de octubre de 2015, en diferentes eventos académicos (Coloquio Arte, Cuerpo y Educación Universidad Santo Tomás, Octubre 16 de 2015; II Foro Distrital de Educación Física en la Biblioteca Virgilio Barco, 28 de octubre de 2015; Simposio Internacional de Formación de Educadores en la Universidad Antonio Nariño, 7 de noviembre de 2015; Encuentro Académico y de Bienestar Redufisica Kennedy 2015 Colegio INEM, 2 Diciembre de 2015), en un segundo momento y durante el primer semestre de 2016 se completa la muestra con otras 25 fotografías (en un mayor tamaño), que rememoran a los pioneros que trabajaron en la consolidación de esta profesión; adicionalmente, se presentan elementos, instalaciones, prácticas y textos que dan cuenta de la formación universitaria de quienes se han forjado como licenciados en esta disciplina pedagógica.

La Gran Exposición Fotográfica Itinerante completa (con las cincuenta fotografías) se estrena durante la Gala de Lanzamiento de la Celebración de los ochenta años de la educación física como profesión en Colombia, el 25 de junio de 2016. Y se exhibe también durante el Conversatorio Pasado-Presente y Futuro de la Educación Física en Colombia, desarrollado en el auditorio del Comité Olímpico Colombiano, el jueves 21 de julio de 2016, y luego en el conversatorio Desempeño Profesional del Licenciado de Educación Física, el 9 de septiembre de 2016 en el hall del salón Presidente del IDRD ${ }^{6}$.

\section{DIÁLOGOS Y ALIANZAS}

Entre diciembre de 2015 y febrero de 2016 se inician diálogos con profesionales del área, los licenciados: Luis Alberto Parra, Olegario Rincón, Martha Moncada, Héctor Peralta, Ana Edurne Camacho, Óscar Ruiz (Jefe Recreación del IDRD), Luis Eduardo Lombo (Coldeportes), Mildrey Mesa (Coldeportes), Baltazar Medina (Presidente del coc) y Diego Corredor (presidente de la ACPEF), y se les presentan las ideas que se tienen para la celebración. De estas gestiones, queda la inquietud de crear un Comité Interinstitucional que

6 Gracias a la alianza con el Centro Virtual de Memoria en Educación y Pedagogía del IDEP, las fotografías se subieron a su página en internet y se pueden apreciar en el siguiente enlace: http://www. idep.edu.co/wp_centrovirtual/?page_id=5785

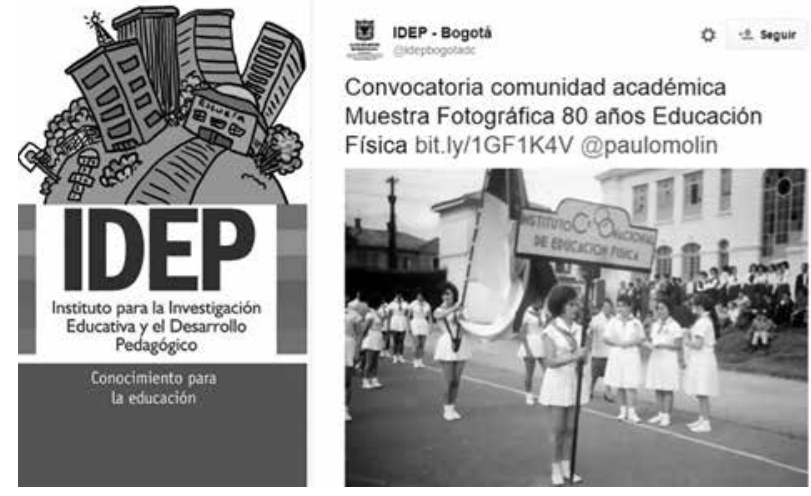

Arte de la Convocatoria hecha a la comunidad académica desde la página del IDEP.
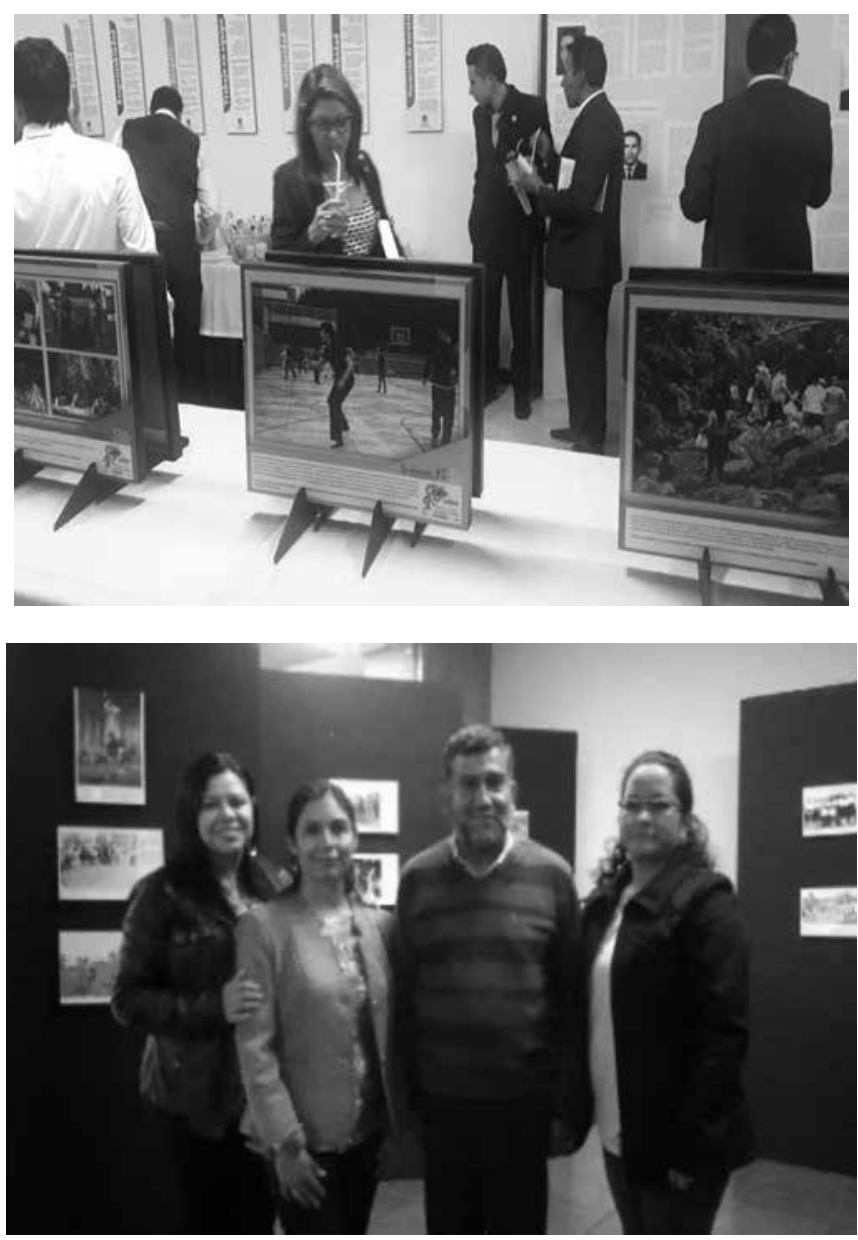

Exhibición de la gran exposición fotográfica itinerante 20152016.

Fuente: Nodo Actividad Física y Cuerpo de la REDDI. 
armonizará una agenda única para no duplicar eventos, esfuerzos ni recursos. El 5 de abril de 2016, en el salón Presidente del IDRD, se crea el Comité Interinstitucional de Conmemoración de los ochenta años de la educación física como profesión en Colombia, integrado por Coldeportes, el Comité Olímpico Colombiano, la Asociación Colombiana de Profesores de Educación Física, el Instituto Distrital de Recreación y Deporte, la Universidad Pedagógica Nacional, la Universidad de Cundinamarca, el Nodo Actividad Física y Cuerpo de la Red Distrital de Docentes Investigadores y un grupo de profesionales que se comprometen a apoyar esta celebración. Se establece que se organizará una Gala de Lanzamiento de la Celebración el sábado 25 de junio de 2016 y que la celebración de esta efemérides abarcará un año, finalizando el 25 de junio de 2017, y que dentro de este periodo se desarrollarán todos los eventos conmemorativos que se organicen, para lo cual cada entidad queda comprometida de informar fechas, lugares y eventos para concretar la agenda. El Nodo Actividad Física y Cuerpo de la REDDI se compromete con la muestra fotográfica, la elaboración de un video y el conversatorio "Pasado, presente y futuro de la educación física en Colombia".

De acuerdo con el compromiso asumido, el Nodo orienta sus acciones a concretar la gran exposición fotográfica itinerante 2016 y el desarrollo del plan de trabajo para la elaboración del video sobre el recorrido histórico de la educación física como profesión en Colombia, aprovechando la alianza con el IDEP y el apoyo y colaboración del comité interinstitucional principalmente de la ACPEF y de la oficina de comunicaciones del Comité Olímpico Colombiano, coordinada por el veterano periodista Alberto Galvis Ramírez. También se trabaja en la elaboración de un logo que identificara la celebración, para lo cual se hace una convocatoria de concurso por parte del Comité de Profesionales de la ACPEF y con el apoyo del Comité Olímpico Colombiano se crea el arte, que aparece al inicio de este artículo.

Como miembros del Comité Interinstitucional de Conmemoración de los ochenta años de la educación física como profesión en Colombia, se participa en varias reuniones de planeación desarrolladas en el Comité Olímpico Colombiano, gracias al total apoyo que se recibe del Presidente, el licenciado Baltazar Medina y, en el Instituto Distrital de Recreación y Deportes (IDRD), con el apoyo del licenciado Óscar Ruiz, para concretar toda la Logística de la Gala de Lanzamiento de la Celebración.

\section{GALA DE LANZAMIENTO DE LA CELEBRACIÓN DE LOS 80 AÑOS DE LA EDUCACIÓN FÍSICA COMO PROFESIÓN EN COLOMBIA}

Bastante nutrida estuvo la imponente ceremonia con la que los Educadores Físicos colombianos, reunidos en el Comité Interinstitucional de Conmemoración de los 80 años de la Educación Física como profesión en Colombia, dieron apertura al año de eventos académicos, sociales, deportivos, investigativos y de educación física, que inicio el sábado 25 de junio de 2016 y se extendió hasta la misma fecha en el año 2017.

La celebración se realizó a partir de las 5 p.m. en las instalaciones del salón Presidente del IDRD, que se adecuó rememorando el ágora griega y desarrolló una variada agenda compuesta por:

- Recepción y bienvenida de participantes, quienes al registrarse recibían un pin botón, con el logo oficial de la celebración a todo color.

- Himno Nacional, interpretado solemnemente por la soprano María Fernanda Romero, del grupo musical de cuerdas clásicas Río.

- Homenaje in memoriam a los educadores físicos que se adelantaron en el camino, mediante un minuto de silencio, distribuido en 30 segundos de total silencio y los restantes de aplausos, liderado por el licenciado Luis Parra, autor del libro 50 años de oro-historia de la educación física en Colombia como profesión 1936-1986.

- Pausa activa, orientada por la estudiante de educación física Luz Mary Rojas, miembro del programa Recreovía del IDRD.

- Palabras de apertura a cargo del magíster Diego Corredor López, presidente de la Asociación Colombiana de Profesores de Educación Física (ACPEF), en representación del Comité Interinstitucional y quien resaltó la importancia de la profesión para el desarrollo del país e invitó a los diferentes actores del área a cerrar filas en torno 


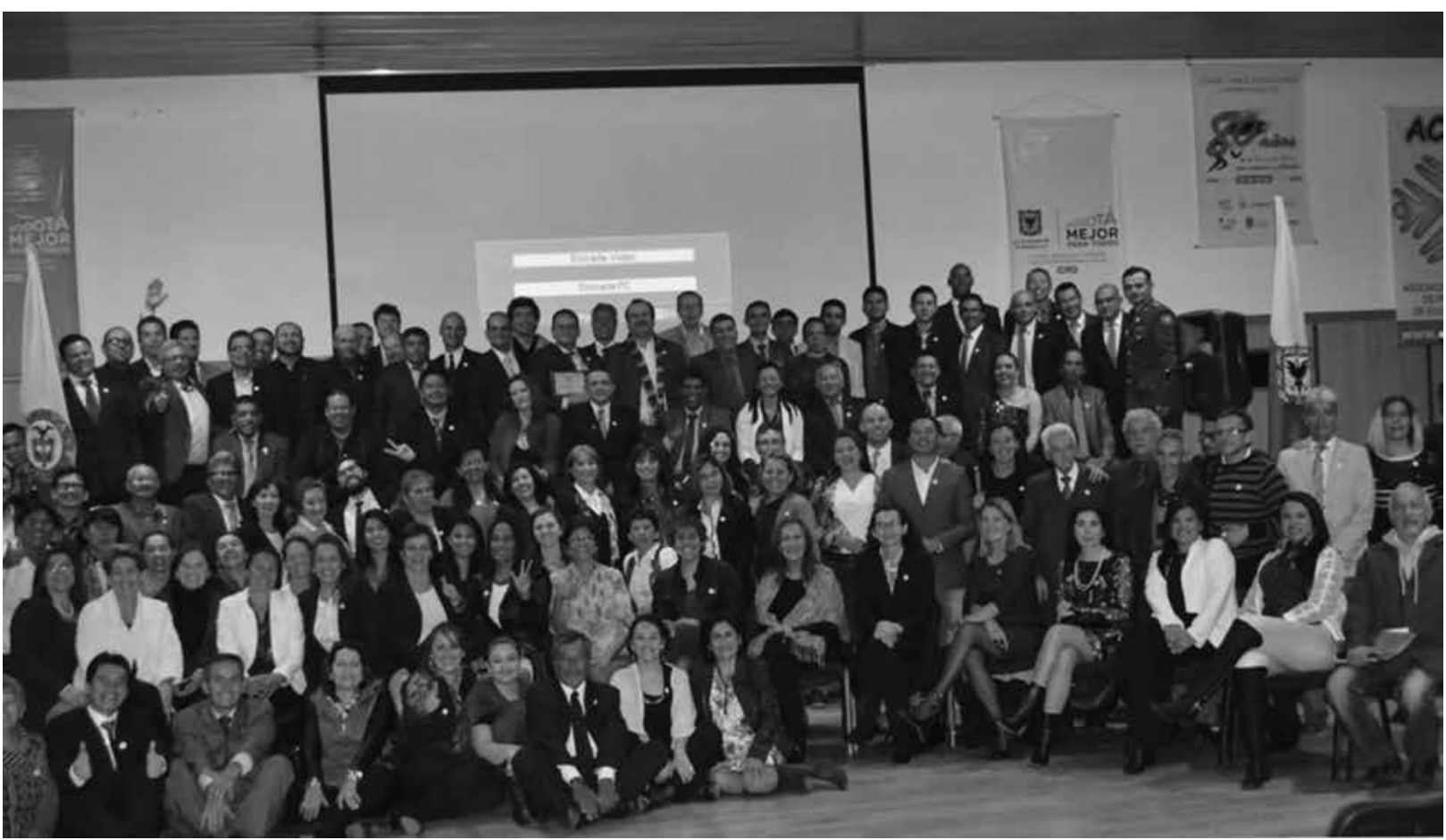

Participantes en la gala de lanzamiento de la celebración de los ochenta años de la Educación Física como Profesión en Colombia. Salón Presidente IDRD, sábado 25 de junio de 2016.

Fuente: Propiedad del Comité Interinstitucional.

a la defensa y consolidación de la educación física como medio ideal para concretar la paz en nuestro país.

- Videos de saludo y de reconocimiento a la profesión, a cargo de la licenciada Martha Moncada de Rojas, destacada educadora y dirigente académica, y el licenciado Baltazar Medina, presidente del Comité Olímpico Colombiano. Igualmente, lo hicieron en forma presencial el licenciado Luis Carlos García, jefe de Bienestar Universitario, y la decana de la Facultad de Educación Física de la Universidad Pedagógica Nacional, Clara Peña, en representación del rector Adolfo León Atehortúa, y finalizó este segmento Mauricio Garzón Camelo, subdirector técnico del IDRD.

- A continuación, se realizó una intervención musical del grupo Río con la Pequeña serenata nocturna de Mozart.

- Se proyectó luego el video sobre el recorrido histórico de la educación física como profesión en Colombia, elaborado por los líderes de las redes (Local de Educación Física de Kennedy y Distrital de Docentes Investigadores, Nodo Actividad Física y Cuerpo).

- Acto seguido, se realiza un sentido homenaje a algunos de los pioneros de la educación física colombiana, quienes con su ejemplo, dedicación y enseñanzas han acompañado la formación de las nuevas generaciones de educadores físicos, en esta ocasión se reconoció la labor de los profesores Martha Moncada de Rojas, Héctor José Peralta Berbesi y Jorge Zabala Cubillos, quienes agradecieron con sentidas palabras este homenaje.

- También se hizo la presentación de la agenda con los eventos que hasta el momento se tienen programados para este año de celebración.

- A continuación, se hace la presentación a la comunidad académica del área de la gran exposición fotográfica itinerante, que se exhibirá en los diferentes eventos de la agenda de este año de celebración, además se resaltó el trabajo investigativo de Historias de Vida, que viene desarrollando el profesor Rafael Morales en 
la UPN. Por último, se invita a unirse al evento multitudinario de Walking Life que se quiere desarrollar a nivel nacional, mediante un recorrido de ocho kilómetros a pie y que involucra a los estudiantes colombianos y sus familias, desplazándose por las principales vías de los municipios y ciudades de Colombia con el apoyo logístico de los profesores de educación física de los entes territoriales y los estudiantes de educación física de las universidades de la región.

- Los profesores de educación física Olga Lucía Moreno y Óscar Mauricio Rincón, del grupo Alma del Tango, realizan una presentación de las obras Tanguera y La comparsita. Se cerró esta parte de la gala con la interpretación del Feliz cumpleaños por parte del grupo Río, entonado con gran motivación y sentimiento por todos y cada uno de los profesionales participantes en la gala.

- Finalmente, se brindó un coctel saludable, que se disfrutó mientras se realizaba la observación de la muestra fotográfica itinerante y se intercambiaba con los otros profesionales de la educación física colombiana asistentes a la Gala; durante este momento se entregó un facsímil del Decreto 1528 del 25 de junio de 1936, mediante el cual se establece la formación profesional de profesores de educación física en Colombia.

Es de resaltar la colaboración y apoyo que en todos los aspectos de organización, logística y protocolo realizaron los licenciados integrantes de la junta de la ACPEF: Daniel López, Floralba Mendoza, María Elisa Cardoso Llamosa, Deyanira Ávila Barón, Nelly Adriana Penagos y Dora Mendoza, quienes fueron respaldados por los profesores Óscar Ruiz, Olegario Rincón, Luis Parra y Nathalia Meneses y un grupo de protocolo de estudiantes de la Universidad Pedagógica Nacional y otro contratado, quienes, con su ardua labor, hicieron que la gala fuera un éxito total y permitiera enaltecer esta efemérides con lujo de detalles. Ofició como maestro de ceremonia el licenciado Leoncio Bravo, quien, con su amplia experiencia en la dirección de este tipo de eventos, engalanó la noche, motivando a todos los participantes a esta sentida ceremonia de exaltación a la profesión de educador físico, en este octogésimo aniversario de acción en nuestro país.
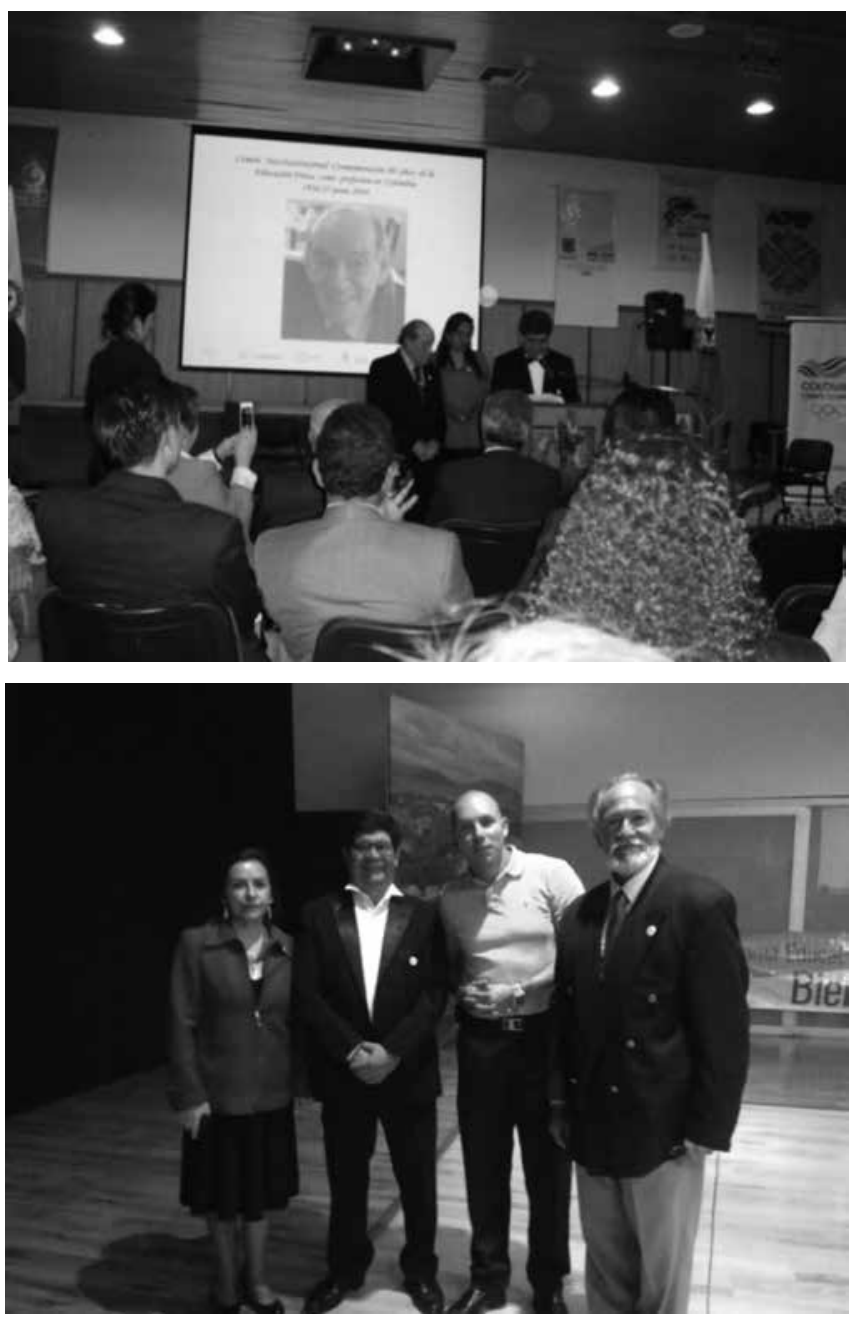

Apartes de la gala y colaboradores en la logística de la ceremonia.

Fuente: Propiedad del Comité Interinstitucional.

\section{CONVERSATORIO "PASADO-PRESENTE Y FUTURO DE LA EDUCACIÓN FÍSICA EN COLOMBIA"}

Con lleno total del auditorio del Comité Olímpico Colombiano, se desarrolló el jueves 21 de julio de 2016 a partir de las siete de la noche el primer evento académico dentro de la programación que se estableció por parte del Comité Interinstitucional. El conversatorio, que brindó a todos y cada uno de los cerca de cien asistentes (estudiantes, dirigentes, investigadores y docentes de colegios, universidades y otros profesionales de diferentes entidades del sector, tanto de la capital como de otras regiones de país) la posibilidad de conocer, dialogar y reflexionar alrededor del tema mencionado, gracias a las 
presentaciones de los panelistas invitados, Mg. Clemencia Anaya Maya (funcionaria de Coldeportes en el cargo de Asesora de Relaciones con Organismos Nacionales e Internacionales y encargada de liderar para Colombia la actualización de la Carta Internacional de la Educación Física, la Actividad Física y el Deporte); Mg. Germán Preciado Mora (docente investigador universitario y de la Secretaría de Educación de Bogotá, Leader Coach de la Red Local de Educación Física de Kennedy y del Nodo Actividad Física y Cuerpo de la Red Distrital de Docentes Investigadores, REDDI, y promotor de la Educación Física Mental y del Walking Life), Mg. Diego Corredor López (docente de la Universidad del Rosario y de la Secretaría de Educación de Bogotá, presidente de la Asociación Colombiana de Profesores de Educación Física, ACPEF), Mg. Pedro Galvis Leal (docente de la Universidad Libre y de la Secretaría de Educación de Bogotá, promotor del Observatorio Pedagógico y Social del Cuerpo de la Secretaría de Educación de Bogotá) y la moderación hecha por la Mg. Nathalia Meneses Runza (colíder de la Red Local de Educación Física de Kennedy del Nodo Actividad Física y Cuerpo de la REDDI), quien propició que los ponentes resolvieran las preguntas orientadoras establecidas para el conversatorio.

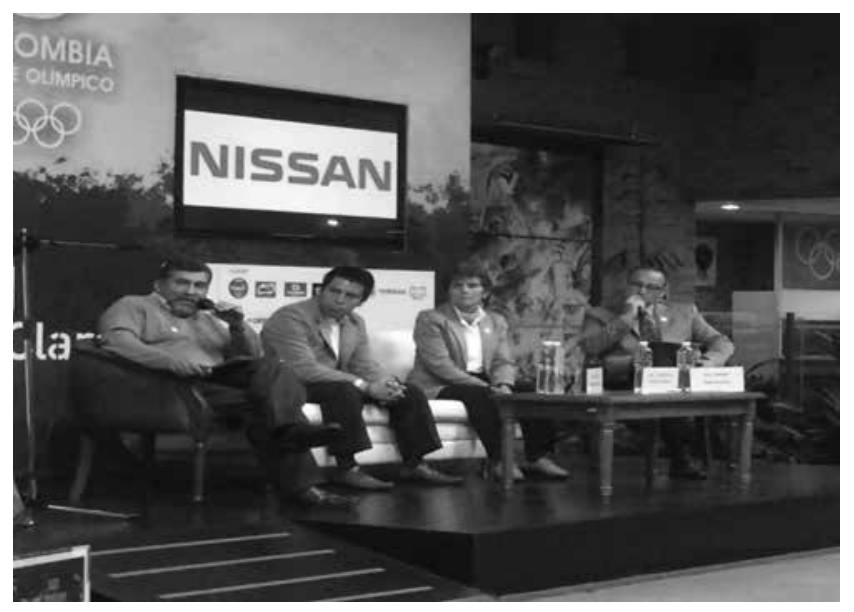

Ponentes del conversatorio "Pasado-presente y futuro de la educación física en Colombia.

Fuente: Nodo Actividad Física y Cuerpo de la REDDI.

Por otra parte, la profesora Clemencia Anaya Maya mostró los antecedentes, los organismos participantes y la logística que se desarrolló en la actualización de la Carta Internacional de la Educación Física, la Actividad Física y el Deporte e invitó a reflexionar sobre la forma en que nuestro país puede aprovechar estos lineamientos para fomentar el desarrollo de la educación física y el deporte en la escuela y en el proceso de formación de nuevos profesionales para estas áreas, desde los organismos gubernamentales y desde las iniciativas privadas. Asimismo, presentó los tres compromisos de la Declaración de Berlín (2013), hecha en la V Conferencia Internacional de Ministros y Altos Dignatarios Responsables de la Educación Física y el Deporte (Mineps V), los cuales están relacionados con: 1. Acceso al Deporte, la Educación Física y la Actividad Física como un derecho fundamental para todos y todas, 2. Promover la inversión en programas de Educación Física y Deporte y 3. Preservar la Integridad en el Deporte frente a la manipulación de Competencias y Corrupción.

Cerró su intervención concluyendo que la Declaración de Berlín y la Carta Internacional de la Educación Física, la Actividad Física y el Deporte deben ser un norte en todos los países de la región de América Latina e invitó a tener en cuenta que, tras la reciente aprobación de la nueva Carta Internacional, en la 38 Conferencia General de Unesco en París (2015), todos los países debemos promocionarla y acogerla en nuestros sistemas nacionales.

Por su parte, el profesor Germán Preciado Mora centró su exposición en mostrar cómo ha sido la evolución de la educación física colombiana en la escuela y en la formación de profesionales para el área en ochenta años de acción profesional. Inició presentando el desarrollo histórico que ha tenido la Educación Física escolar en 208 años de acción en nuestro país, dado que situó en el 31 de julio del año 1808 la primera referencia a la educación física, hecha por el abogado payanés Francisco Antonio de Ulloa en su ensayo "Sobre el influxo del clima en la educación física y moral del hombre del Nuevo Reino de Granada", publicado en cerca de diez entregas desde el número 31 en el Semanario del Nuevo Reino de Granada, dirigido por el sabio, periodista y prócer neogranadino Francisco José de Caldas.

Posteriormente, continuó mostrando la evolución de la educación física escolar en cuanto a las tendencias, los objetivos, la orientación metodológica y los principales trabajos con los estudiantes, que se planteó en las diferentes reformas acaecidas a lo largo del siglo XIX, desde las promulgadas por el General Francisco de Paula Santander en 1826 y 1827 y por El Libertador Simón Bolívar en 1829 y luego las de los años 1844, 1871 y 1988, hasta llegar a la Ley 80 de 1925, cuando 
la educación física de la escuela se asume como una política del Estado.

Abordó luego los cambios originados en el periodo 1936 a 1950, con la creación del Instituto Nacional de Educación Física (Inef) el jueves 25 de junio de 1936, mediante el Decreto 1528, al que se le encargó, y efectivamente asumió, la formación profesional de profesores para la educación física, que tuvo su primera cohorte de doce profesores el 21 de diciembre de 1937. Cerró esta parte de la exposición con los principales aspectos de lo acaecido entre 1951 a 1996, cuando se terminó de reglamentar la legislación que rige actualmente la educación física escolar, después de la promulgación de la nueva Constitución Política de Colombia en 1991, con las siguientes normas: la Ley 60 de 1993, la Ley 115 de 1994, el Decreto 1860 de 1995, la Ley 181 de 1995 y la Resolución 2343 de 1996.

Adicionalmente, el Mg. Germán Preciado mostró los aspectos más relevantes (sitios de formación, tiempo requerido, titulaciones e instituciones responsables) de la formación de profesionales para la Educación Física, desde 1936 hasta nuestros días. Actualmente, se cuenta con 84 diferentes programas en 48 Instituciones de educación superior y 137 escuelas normales superiores, distribuidas por 36 municipios y ciudades de nuestra geografía nacional. Culminó su exposición invitando a los asistentes a ampliar la información, consultando la "Evolución histórica de la educación física escolar", que se describe en el libro de los Lineamientos curriculares de la educación física, recreación y deportes, y a observar el video 80 años de la educación física como profesión en Colombia 7 .

Por otra parte, el profesor Diego Corredor López mostró cómo la educación física escolar en la actualidad se debe mirar bajo tres aspectos: desde lo pedagógico, como una oportunidad legal y como un campo de estudio. Desde lo pedagógico, se debe tener en cuenta la autonomía que las instituciones tienen desde el PEI para manejar una educación física que se orienta desde un modelo pedagógico, un currículo, unas didácticas y unos procesos evaluativos, de acuerdo con el tipo de estudiantes que a ella asisten y que buscan formar como fruto del proceso educativo. Como oportunidad legal, debe armonizar lo establecido en la Constitución Política de Colombia,

7 https://www.youtube.com/watch?v=uFn5pTbQmg0. con lo legislado en las leyes 115 de Educación y 181 del Deporte, en busca de una educación física de calidad para los niños y jóvenes escolares. Y como campo de estudio, a través de la relación interdisciplinaria que tiene la educación física con las demás áreas obligatorias del currículo y los aportes y formas que puede utilizar en su acción pedagógica, como son el juego, la recreación, la expresión corporal, la actividad física y el deporte. Luego, el profesor Diego aborda la relación que la educación física tiene como área de intersección, en la interacción que se presenta en la escuela, de los campos de la educación, la salud y el deporte, en busca de ofrecer a los estudiantes la posibilidad de alcanzar las competencias que les permitan delinear un proyecto de vida; que les ayude en su salud, bienestar y calidad de vida y en el desarrollo de una disciplina hacia el trabajo físico que conserven durante toda su vida. En cuanto a la formación de profesionales del área, enfatizó sobre la importancia de reconocer las diferencias en los procesos formativos, en los diferentes niveles de nuestro sistema de formación profesional y su relación con los campos de acción de los egresados, y cómo se debe tratar de armonizar y consensar esta situación para que no se presenten dificultades que generen problemáticas que afecten a la profesión y a los usuarios de la acción profesional. Finalmente, concluyó que la tarea y el reto es reconocernos como colegas y potencializar las lecciones aprendidas en beneficio de la población colombiana que demanda los servicios del campo de la educación física.

Por último, el profesor Pedro Galvis Leal postuló la pregunta: ¿Cómo debe ser el futuro de la educación física colombiana, en la escuela y en la formación de profesionales para el área, de cara al acuerdo de paz? Esto a partir de la lectura de un escrito titulado "Por una heterotopía del cuerpo. Una decisión por las experiencias diferenciadas, espacios otros, tiempos otros", que inicia con la reflexión de Michel Foucault en Les mots et les choses (1966):

Las utopías consuelan: porque, aunque no tengan un lugar real, se despliegan, sin embargo, en un lugar maravilloso y liso; abren ciudades, avenidas, jardines bien plantados, países benignos, aun cuando su acceso sea quimérico. Las heterotopías inquietan, sin duda, porque gazapan secretamente el lenguaje, porque impiden nombrar esto y aquello, porque quiebran los nombres comunes o los encabalgan, porque arruinan de antemano su sintaxis (...). Las utopías permiten las fábulas (...), las 